Revista Brasileira de Agricultura Irrigada v.9, nº.6, p.392 - 399, 2015

ISSN 1982-7679 (On-line)

Fortaleza, CE, INOVAGRI - http://www.inovagri.org.br

DOI: $10.7127 /$ rbai.v9n600325

Protocolo 325.15 - 27/06/2015 Aprovado em 10/11/2015

\title{
EVAPOTRANSPIRAÇÃO E COEFICIENTE DE CULTURA PARA MELANCIA
}

Rodrigo Rafael da Silva ${ }^{1}$, Irai Manuela Santana Santos ${ }^{2}$, Gertrudes Macário de Oliveira ${ }^{3}$, Adheilton Rogers Pilé de Carvalho ${ }^{4}$, Pedro Pereira Santos Júnior ${ }^{5}$, Iris Santana Gonçalves ${ }^{6}$

\section{RESUMO}

Considerando que o conhecimento das reais necessidades hídricas das culturas e o manejo correto da irrigação permite fornecer às plantas a quantidade ideal de água que promova o desenvolvimento adequado e a expressão de todo seu potencial produtivo, o presente trabalho teve como objetivo, determinar a evapotranspiração e os coeficientes de cultura para os diferentes estádios de desenvolvimento da melancia. A pesquisa foi conduzida de dezembro de 2014 a fevereiro de 2015 no campo experimental do DTCS/UNEB em Juazeiro. A cultivar utilizada foi a Sugar Baby. A evapotranspiração da cultura (ETc) foi obtida a partir de leituras diárias em evapotranspirômetros e a evapotranspiração de referência (ETo), obtida pelo método de Penman-Monteith. O coeficiente de cultura foi determinado pela relação Kc = ETc/ETo. Os maiores valores da ETc foram observados no estádio intermediário, atingindo valores máximos de até $10,4 \mathrm{~mm} \mathrm{dia}{ }^{-1}$. A ETc média para todo o ciclo da cultura foi de 5,4 $\mathrm{mm} \operatorname{dia}^{-1}$. Os valores de Kc variaram de 0,51 para o estádio inicial a 1,23 para o intermediário. A demanda atmosférica foi determinante para o valor de Kc observado no estádio II, demonstrando a importância da calibração do Kc para condições climáticas específicas.

Palavras-chave: requerimento hídrico da cultura, evapotranspiração de referência, condições climáticas.

\section{EVAPOTRANSPIRATION AND CROP COEFFICIENT FOR WATERMELON}

\footnotetext{
ABSTRACT

Whereas knowledge of the real water needs of crops and the correct management of irrigation allows supply to plants the optimum amount of water that promotes appropriate development and expression of all their productive potential, the present work had as objective to determine the evapotranspiration and crop coefficients for the different developmental stages

${ }^{1}$ Acadêmico de Engenharia Agronômica, Bolsista CNPq, DTCS/UNEB, Av. Edgard Chastinet, s/n, Bairro São Geraldo, 48900-000, Juazeiro, BA, Brasil. Fone: (74) 3611 7362. Email: rodrigosilva_rafael@hotmail.com

${ }^{2}$ Mestra em Horticultura Irrigada, PPHI/DTCS/UNEB, Juazeiro, BA. Email: irai.manuela@gmail.com

${ }^{3}$ Doutora, Professora do DTCS/UNEB, Juazeiro, BA. Email: gemoliveira@uneb.br

${ }^{4}$ Acadêmico de Engenharia Agronômica, DTCS/UNEB, Juazeiro, BA. Email: adheilton@hotmail.com

${ }^{5}$ Acadêmico de Engenharia Agronômica, Bolsista CNPq, DTCS/UNEB. Juazeiro, BA. Email: ppereirasj@hotmail.com

${ }^{6}$ Acadêmica de Engenharia Agronômica, Bolsista FAPESB, DTCS/UNEB, Juazeiro, BA. Email: irissg@hotmail.com
} 
of the watermelon. The survey was conducted December 2014 to February 2015 in the experimental field of the DTCS/UNEB in Juazeiro. The cultivar used was the Sugar Baby. The crop evapotranspiration (ETc) was obtained from daily readings in evapotranspirometers and the reference evapotranspiration (ETo), obtained by the Penman-Monteith method. The crop coefficient was determined by the ratio $\mathrm{Kc}_{\mathrm{c}}=\mathrm{ETc} / \mathrm{ETo}$. The highest values of ETc were observed in the intermediate stage, reaching maximum values of up to $10.4 \mathrm{~mm} \mathrm{day}^{-1}$. The average ETc for the entire cycle of culture was $5.4 \mathrm{~mm} \mathrm{day}^{-1}$. The Kc values ranged from 0.51 to the initial stage the 1.23 for the intermediary. The atmospheric demand determined the Kc value observed in stage II, demonstrating the importance of Kc calibration to specific climatic conditions.

Keywords: water requirement of culture, reference evapotranspiration, climatic conditions.

\section{INTRODUÇÃO}

A melancia (Citrullus lanatus), pertence à família das cucurbitáceas, e é uma das principais olerícolas encontradas em todo o mundo. De acordo com dados da FAOSTAT (2015), em 2012, os maiores produtores mundiais de melancia foram a China (70 t ha $\left.{ }^{-1}\right)$, o Peru $\left(40,44 \mathrm{t} \mathrm{ha}^{-1}\right)$ e o Brasil, ocupando a quarta posição com 20,79 tha $^{-1}$.

As áreas plantadas ainda são de melancias grandes, com peso médio acima de $6 \mathrm{~kg}$. Recentemente, destaca-se o surgimento de novos tipos de melancias, as chamadas mini melancias, com peso entre 1 e $4 \mathrm{~kg}$. Isto se deve, principalmente, à exigência do mercado por produtos alternativos, onde o consumidor prefere frutos menores e de qualidade.

A melancia, apesar de ser uma cultura resistente à seca, para a obtenção de altas produtividades, a prática da irrigação é indispensável e tem sido utilizada por muitos produtores sem o devido conhecimento (MAROUELLI et al., 2012). Esses autores destacam que a cultura é bastante exigente no manejo da aplicação de água. E, sabe-se que, para uma reposição de água eficiente, o uso de indicadores das necessidades hídricas da cultura torna-se imprescindível.

Segundo Carvalho et al. (2007), para um manejo eficiente da irrigação, de qualquer que seja a cultura, é essencial a determinação da evapotranspiração. Silva \& Marouelli (1998) colocam que, para o manejo adequado da água de irrigação é necessário o controle da umidade do solo e/ou o conhecimento da evapotranspiração durante todo o ciclo da cultura. Para tanto, é indispensável que os parâmetros relacionados às plantas, ao solo e ao clima, sejam conhecidos para que se possa determinar o momento oportuno de irrigar e a quantidade de água a ser aplicada.

A forma usual de se quantificar a água a ser aplicada ao longo do ciclo de uma cultura é considerar os processos de evaporação do solo e de transpiração da planta, conjuntamente, denominado de evapotranspiração. A evapotranspiração da cultura (ETc) é uma variável básica da irrigação e depende dos elementos meteorológicos, da cultura e do solo podendo ser medida diretamente (lisímetros) ou indiretamente (equações combinadas) por vários métodos (CHAVES et al., 2005).

Diante da dificuldade de obtenção da medida direta da ETc, tem-se recorrido à utilização de equações empíricas, por serem mais práticas e viáveis de serem usadas para fins de manejo da irrigação. De acordo com Doorenbos \& Pruitt (1977), a ETc pode ser calculada a partir da evapotranspiração de referência (ETo) e do coeficiente de cultivo (Kc) em seus diferentes estádios fenológicos. O Kc é determinado empiricamente e varia com a cultura, com seu estádio de desenvolvimento, com o clima e com as práticas agronômicas adotadas. Para o planejamento racional da irrigação é de 
fundamental importância o conhecimento da ETc e do Kc durante os estádios de desenvolvimento da cultura (ANDRADE et al., 2013).

Diversos têm sido os estudos voltados para a determinação de coeficientes de cultura. Entretanto, Doorenbos \& Pruitt (1977) enfatizam a necessidade de calibração dos coeficientes de cultura sob condições climáticas específicas. Allen et al. (1998), recomendam que os valores de Kc e da duração dos estádios da cultura sejam ajustados por meio de experimentos para cada região, de acordo com a variedade plantada, condições climáticas e as técnicas de cultivo utilizadas. Silva et al. (2006) colocam que, o conhecimento do coeficiente de cultura é fundamental para

\section{MATERIAIS MÉTODOS}

A pesquisa foi conduzida no período de dezembro de 2014 a fevereiro de 2015 no campo experimental do Departamento de Tecnologia e Ciências Sociais - DTCS da Universidade do Estado da Bahia UNEB, em Juazeiro (Lat. 09²4'50”'S, Long. 40³0’10”W, alt. $368 \mathrm{~m}$ ). O solo da área experimental, de acordo com o Laboratório de Análises de Solo, Água e Calcário - LASAC do DTCS/UNEB foi classificado como Neossolo Flúvico.

As mudas de melancia foram produzidas em casa de vegetação com $50 \%$ de sombreamento, semeadas em bandejas de polipropileno no dia 09 de dezembro e transplantadas no dia 27 de dezembro de 2014. A cultivar utilizada foi a Sugar baby. Na área, a cultura foi implantada sobre se outorgar, dimensionar e manejar a irrigação de uma cultura e, uma vez que esses valores variam conforme a disponibilidade energética do local, variedade e idade da planta é importante à obtenção de valores regionalizados. Ferreira et al. (2011), destacam que a utilização de valores obtidos em outras regiões pode gerar erros consideráveis na estimativa do consumo hídrico da cultura.

Diante do exposto, considerando a carência de informações quanto às necessidades hídricas da melancia no Vale do São Francisco, o presente trabalho teve como objetivo, determinar a evapotranspiração e os coeficientes de cultura para os diferentes estádios de desenvolvimento da melancia na região de Juazeiro,

BA.

camalhões, com espaçamento de 2 m entre linhas e $0,5 \mathrm{~m}$ entre plantas; irrigada por sistema localizado, com gotejos espaçados em $0,5 \mathrm{~m}$.

Para a determinação da evapotranspiração da cultura (ETc) foram realizadas leituras diárias em dois evapotranspirômetros de lençol freático constante $\left(5,0 \mathrm{~m}^{2}\right.$ e $1,30 \mathrm{~m}$ de profundidade), instalados no centro da área experimental. A evapotranspiração de referência (ETo) foi determinada com base nos dados obtidos na estação meteorológica automática localizada em frente a área experimental utilizando o método de Penman-Monteith parametrizado pela FAO (Allen et al., 1998).

$$
E T o=\frac{0,408 \Delta(R n-G)+\frac{\gamma 900 U_{2}\left(e_{s}-e_{a}\right)}{t+273}}{\Delta+\gamma\left(1+0,34 U_{2}\right)}
$$

em que:

$\mathrm{Rn}$ - radiação líquida total diário $\left(\mathrm{MJ} \mathrm{m}^{-2} \mathrm{~d}^{-}\right.$ $1)$;
$\mathrm{G}$ - fluxo de calor no solo $\left(\mathrm{MJ} \mathrm{m}^{-2} \mathrm{~d}^{-1}\right)$;

$\gamma$ - parâmetro psicrométrico $\left(\mathrm{kPa}^{\circ} \mathrm{C}^{-1}\right)$; 
$\mathrm{U}_{2}$ - velocidade do vento a $2 \mathrm{~m}$ de altura (m

$\left.\mathrm{s}^{-1}\right)$;

es - pressão de saturação de vapor $(\mathrm{kPa})$;

ea- pressão parcial de vapor $(\mathrm{kPa})$;

$\mathrm{T}$ - temperatura média do ar $\left({ }^{\circ} \mathrm{C}\right)$;

$\Delta$ - declividade da curva de pressão de vapor em relação à temperatura do ar $(\mathrm{kPa}$ ${ }^{\circ} \mathrm{C}^{-1}$ ).

O coeficiente de cultura (kc) foi determinado pela relação entre a evapotranspiração da cultura (ETc) e a evapotranspiração de referência (ETo):

\section{RESULTADOS E DISCUSSÃO}

Com base no acompanhamento dos estádios fenológicos da melancia, determinou-se o número de dias de cada fase da cultura (Tabela 1). As durações diferiram das encontradas por Miranda et al. (2004), trabalhando com a variedade Crimson Sweet na região de Paraipaba,

$$
K c=\frac{E T c}{E T o}
$$

O período de desenvolvimento da cultura foi dividido em quatro estádios: Inicial (I) - do transplante das mudas até o estabelecimento inicial $(10 \%$ do crescimento vegetativo); Vegetativo (II) do estabelecimento inicial até o início da floração; Floração (III) - do início da floração até o início da maturação dos frutos; Maturação (IV) - do início da maturação até a colheita.

CE, 23, 14, 19 e 14 dias para os períodos inicial, de crescimento, intermediário e final, respectivamente. O número total de dias do ciclo da cultura do transplantio até a colheita foi de 60 dias, valor próximo ao determinado para a mini melancia (65 a 100 dias após o transplante das mudas), como citado por Campagnol et al. (2012).

Tabela 1. Duração de cada estádio fenológico (dias) da cultura, média da evapotranspiração da cultura (ETc), média da evapotranspiração de referencia (ETo) e coeficientes de cultura (Kc) para as diferentes fases de desenvolvimento da melancia. Juazeiro, BA, 2015.

\begin{tabular}{cccccc} 
Estádios & $\begin{array}{c}\text { Duração } \\
(\text { dias })\end{array}$ & $\begin{array}{c}\text { ETc } \\
\left(\mathrm{mm} \mathrm{dia}^{-1}\right)\end{array}$ & $\begin{array}{c}\text { ETo } \\
\left(\mathrm{mm} \mathrm{dia}^{-1}\right)\end{array}$ & Kc & $\begin{array}{c}\text { Kc } \\
\text { Kossam, 1979) }\end{array}$ \\
\hline I - Inicial & 9 & 3,0 & 5,9 & 0,51 & $0,40-0,50$ \\
II - & 15 & 3,8 & 7,3 & 0,52 & $0,70-0,80$ \\
$\begin{array}{c}\text { Vegetativo } \\
\text { III - }\end{array}$ & 26 & 7,9 & 6,4 & 1,23 & $0,95-1,05$ \\
$\begin{array}{c}\text { Floração } \\
\text { IV - }\end{array}$ & 10 & 6,9 & 6,1 & 1,13 & $0,65-0,75$ \\
Maturação & & - & - & - & - \\
\hline Total & 60 & & &
\end{tabular}

Ainda na Tabela 1, é possível observar que a menor demanda hídrica da cultura foi observada durante os estádios I e II. Tal fato se explica, porque nesse período, a planta encontrase na fase inicial do seu desenvolvimento, e devido à menor área foliar, o principal fator que rege a perda hídrica é a evaporação da água na superfície do solo.
A partir do início do florescimento, houve um maior consumo de água acompanhando o rápido desenvolvimento da cultura. O período de maior demanda hídrica da melancia ocorreu durante a fase de enchimento dos frutos e máximo desenvolvimento vegetativo, sendo observados valores máximos de evapotranspiração da cultura diária de até 10,4 mm dia ${ }^{-1}$. Para todo o período, a 
média foi de $5,4 \mathrm{~mm} \operatorname{dia}^{-1}$ e o total de 323,5 mm.

Segundo Doorenbos e Kassam (1979), a necessidade hídrica da melancia varia de 400 a $600 \mathrm{~mm}$, para um ciclo total de 100 dias. $O$ total acumulado durante 0 presente experimento foi inferior ao descrito pelos autores, porém, superior ao encontrado por Carvalho et al. (2007), 277 $\mathrm{mm}$ para o ciclo da melancia sem sementes. Essas diferenças podem ser explicadas em função da duração do ciclo da cultura e pelas condições edafoclimáticas da região.

Comparando os coeficientes de cultura (Kc) obtidos para as condições do experimento, com aqueles propostos por Doorenbos e Kassam (1979) para a cultura da melancia (Tabela 1), observa-se que com exceção do estádio inicial, em que o valor do Kc $(0,51)$ foi bem próximo ao do limite superior indicado por Doorenbos e Kassam (1979) para a cultura $(\mathrm{Kc}=0,50)$, para os demais estádios, os valores de Kc apresentados por Doorenbos e Kassam superestimaram no estádio vegetativo (II) e subestimaram as necessidades hídricas da cultura nos estádios de floração (III) e maturação (IV). O valor do Kc encontrado no estádio II $(0,52)$ representou cerca de
$74,3 \%$ do valor do Kc mínimo $(0,70)$ apresentado por Doorenbos e Kassam (1979) para esse estádio. Isso se justifica, devido a alta demanda atmosférica, como pode ser comprovado pelo elevado valor da evapotranspiração de referência (ETo) nesse período, 7,3 $\mathrm{mm}$.

Os valores de Kc encontrados neste estudo diferiram dos encontrados por Carvalho et al. (2007), para a melancia sem sementes irrigada por gotejamento, sob as condições edafoclimáticas de Fortaleza, cujos valores foram de 0,38, 0,52; 0,98 e 0,59 , para os estádios inicial, vegetativo, intermediário e final, respectivamente.

Bastos et al. (2012), ao determinar o coeficiente de cultura para a melancia no litoral do Piauí, encontraram para a fase intermediária, valor de Kc igual a 1,30. Essas diferenças entre os Kcs encontrados nesse trabalho e os propostos na literatura podem ser atribuídas, à variedade utilizada e às condições locais do desenvolvimento da cultura, revelando a importância de se calibrar os coeficientes para condições específicas.

As condições climáticas registradas para cada estádio de desenvolvimento da cultura são apresentadas na Tabela 2.

Tabela 2. Temperatura média do ar (Tar), radiação solar global (Rg), umidade relativa do ar (\%), velocidade do vento ( $\mathrm{Vv})$ e precipitação pluviométrica para cada estádio de desenvolvimento da cultura da melancia. Juazeiro, BA, 2015.

\begin{tabular}{cccccc}
\hline Estádios & $\begin{array}{c}\text { Tar } \\
\left({ }^{\circ} \mathrm{C}\right)\end{array}$ & $\begin{array}{c}\mathrm{Rg} \\
\left(\mathrm{MJ} \mathrm{m}^{2} \mathrm{~d}^{-1}\right)\end{array}$ & $\begin{array}{c}\text { UR } \\
(\%)\end{array}$ & $\begin{array}{c}\text { Vv } \\
\left(\mathrm{m} \mathrm{s}^{-1}\right)\end{array}$ & $\begin{array}{c}\text { Precipitação } \\
(\mathrm{mm})\end{array}$ \\
\hline I & 26,3 & 22,6 & 57,3 & 2,4 & 0,0 \\
II & 27,8 & 24,0 & 49,0 & 3,3 & 0,0 \\
III & 27,4 & 21,2 & 55,2 & 2,5 & 21,4 \\
IV & 26,3 & 19,2 & 62,9 & 2,7 & 6,9 \\
\hline
\end{tabular}

Em média, os valores de temperatura ficaram na faixa ideal para o desenvolvimento da melancia, variando de 26,3 ${ }^{\circ} \mathrm{C}$ (estádios I e IV) a $27,8{ }^{\circ} \mathrm{C}$ (estádio II). Rezende et al. (2010), afirmam que as cucurbitáceas se adaptam bem às zonas quentes e semiáridas, com alta luminosidade e temperaturas do ar entre 18
${ }^{\circ} \mathrm{C}$ e $30^{\circ} \mathrm{C}$, não tolerando temperaturas abaixo de $10^{\circ} \mathrm{C}$.

Observa-se que apesar do estádio II ter apresentado valores elevados de temperatura do ar, radiação solar, velocidade do vento e baixa umidade relativa do ar, condições climáticas propícias para maior perda d’água para a 
atmosfera, os valores de ETc não foram tão elevados (Tabela 1), devido a menor área foliar da cultura nesse estádio. Observa-se ainda na Tabela 2, a ocorrência de precipitação pluviométrica nos estádios III e IV; essa precipitação foi distribuída em cinco dias do estádio III e dois dias do estádio IV.

Segundo Souza et al. (2008), dentre os fatores climáticos, a precipitação é determinante para definição da época de plantio e da forma de cultivo, além disso, o excesso de chuvas durante 0 desenvolvimento da cultura aumenta a incidência de doenças.

A Figura 1 apresenta a evolução do coeficiente de cultura e do déficit de saturação de vapor do ar ao longo do ciclo de desenvolvimento da melancia.

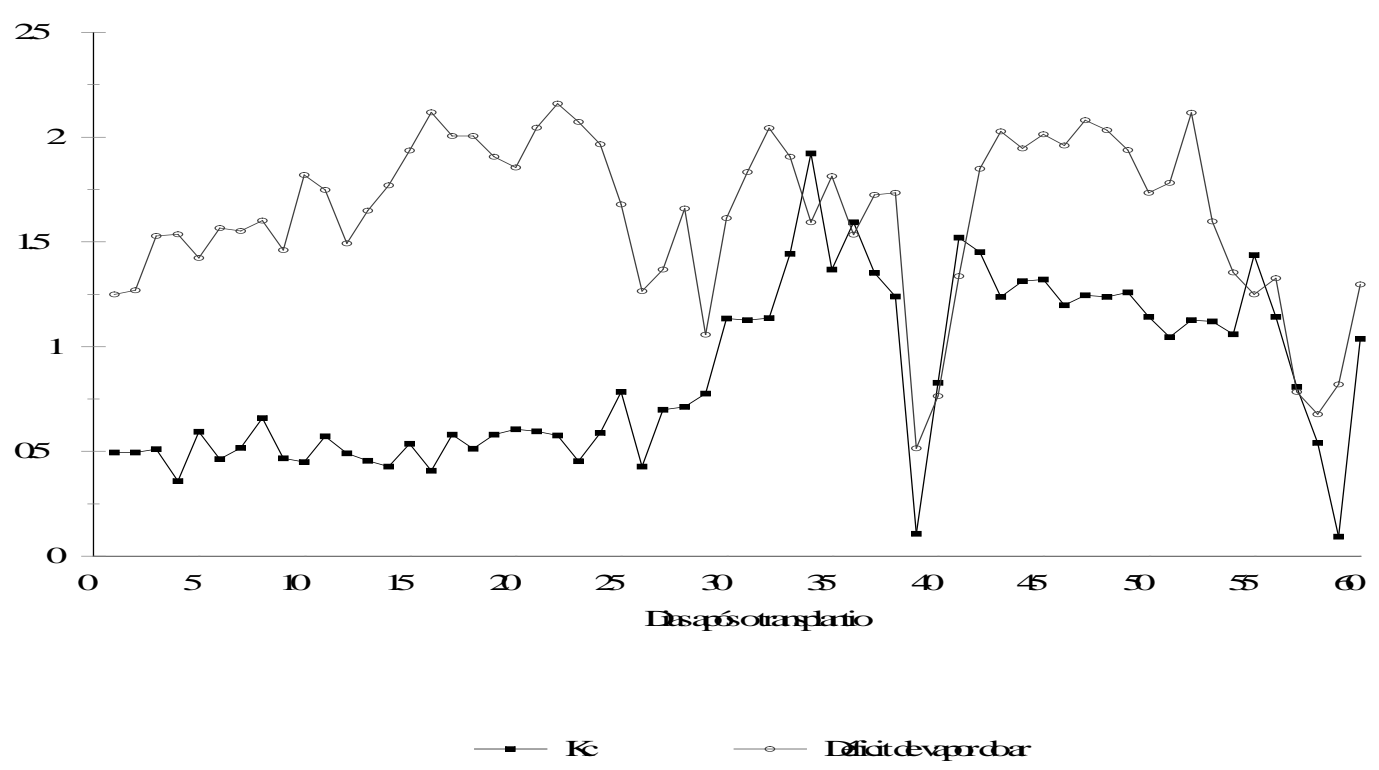

Figura 1. Evolução do coeficiente de cultura e do déficit de saturação de vapor do ar ao longo do ciclo de desenvolvimento da melancia.

Verifica-se na Figura 1, o aumento do Kc com o aumento do índice de área foliar e formação do fruto e consequente redução, no período de maturação. Constata-se ainda, a alta demanda atmosférica no estádio II, em que o déficit de saturação de vapor do ar atingiu valores máximos de até 2,16 kPa. A ocorrência de precipitação nos estádios III e IV pode ser observada na Figura 1, pela diminuição do déficit de saturação de vapor do ar e consequentemente, do coeficiente de cultura.
Os maiores valores da evapotranspiração da cultura (ETc) foram observados no estádio intermediário, atingindo valores máximos de até $10,4 \mathrm{~mm}$ dia $^{-1}$. A ETc média para todo o ciclo da cultura foi de $5,4 \mathrm{~mm} \mathrm{dia}^{-1}$.

Os valores de Kc variaram de 0,51 para o estádio inicial a 1,23 para o intermediário.

A demanda atmosférica foi determinante para o valor de Kc observado no estádio II, demonstrando a importância da calibração do Kc para condições climáticas específicas. 
ALLEN, R.G.; PEREIRA, L.S.; RAES, D.; SMITH, M. Crop evapotranspiration: guidelines for computing crop water requirements. Roma: FAO, 1998. 301p. (Irrigation and Drainage Paper, 56).

ANDRADE, A. R. S. de; CRUZ, A. F. da S.; CAVALCANTE, E. C. dos S.; ALBUQUERQUE, J. C. F. de; SOUZA, W. M. de. Estimativa da evapotranspiração e dos coeficientes de cultura para diferentes fases de desenvolvimento da melancia. Revista Brasileira de Geografia Física, v.6, n.5, p.1417-1429. 2013.

BASTOS, E; SILVA, C. R; RODRIGUES, B. H. N; ANDRADE JR, A. S; IBIAPINA, L. M. M. Evapotranspiration and crop coefficient of drip irrigated watermelon in Piauí coastline, Brazil, Engenharia Agrícola, Jaboticabal, v.32, n.3, p.582590, 2012.

CAMPAGNOL, R; JUNQUEIRA, R. P. D; MELLO, S. C; Cultivo de mini melancia em casa de vegetação. Cartilha Técnica Piracicaba: USP/ESALQ/Casa do Produtor Rural, p.56, 2012.

CARVALHO, L. C. C.; BEZERRA, F. M. L.; CARVALHO, M. A. R. Evapotranspiração e coeficientesde cultivo da melancia sem sementes. Revista Ciência Agronômica, v.39, n.01, p.53 59, 2007.

CHAVES, S. W. P.; AZEVEDO, B. M. DE; MEDEIROS, J. F. DE; BEZERRA, F. M. L.; MORAIS, N. B. de. Evapotranspiração e coeficiente de cultivo da pimenteira em lisímetro de drenagem. Revista Ciência Agronômica, v.36, p.262-267, 2005.

DOORENBOS, J.; PRUITT, W. O. Guidelines for predicting crop water requirements. Rome : FAO, 1977. 194p. (FAO.Irrigation and Drainage Paper, 24).
DOORENBOS, J.; KASSAM, A. H. Yield response to water.Rome: FAO, 1979. 193p. (FAO. Irrigation and Drainage Paper, 33).

FAOSTAT - Food and Agriculture Organization of the United Nations. Production, crops. Disponível em $<$ http://faostat.fao.org/site/339/default.aspx $>$ Acesso em 15 Abr. 2015.

FEREIRA, V. M; JUNIOR, A. A; KLAR, A. E; MORAIS, E. L. C; BRAGA, D. L.Coeficientes de cultura para a melancia irrigada por gotejamento. In: CONGRESSO BRASILEIRO DE ENGENHARIA AGRICOLA, 40. 2011. Cuiabá. Geração de tecnologias inovadoras e o desenvolvimento do cerrado brasileiro: anais. Cuiabá: SBEA, 2011.

MAROUELLI, W.; BRAGA, M. B.; ANDRADE JÚNIOR, A. S. de. Irrigação na cultura da melancia. Circular Técnica, 108. Ministério da Agricultura, Pecuária e Abastecimento, Brasília, DF, 2012.

MIRANDA, F. R; OLIVEIRA, J. J. G; SOUZA, F. Evapotranspiração máxima e coeficientes de cultivo para a cultura da melancia irrigada por gotejamento. Revista Ciência Agronômica, V.35, n.1, p.36 43, 2004.

REZENDE, G. M. de; DIAS, R. de C. S.; COSTA, N. D. Cima. In: DIAS, R. de C. S.; REZENDE, G. M. de; COSTA, N. D. (Ed.), Sistema de produção de melancia. Petrolina: Embrapa semiárido, 2010. (Embrapa semiárido. Sistemas de Produção 6) Disponível em: http://sistemasdeproducao.cnptia.embrapa. br/FontesHTML/Melancia/SistemaProduca oMelancia/clima.htm. Acesso em: 22 mai. 2015.

SILVA, C. M.; ALVES JÚNIOR, J.; SILVA, T. J. A.; FOLEGATTI, M. V.; CAMPECHE, L. F. S. M.; Variação sazonal na evapotranspiração de plantas jovens de lima ácida 'tahiti'. Irriga, Botucatu, v. 11, n. 1, p. 26-35, 2006. 
Silva et al.

SILVA, W. L. C.; MAROUELLI, W. A. Manejo de irrigação em hortaliças no campo e em ambientes protegidos. In: FARIA, M.A. (Coord.). Manejo de irrigação. Lavras: UFLA/SBEA, 1998. p. 311-348.
SOUZA, J. O.; GRANGEIRO, L. C.; SANTOS, G. M.; COSTA, N. D.; SANTOS, C. A. F.; NUNES, G. H. S. Avaliação de genótipos de cebola no semiárido Nordestino. Horticultura Brasileira, v.26, p.97-101, 2008. 